\title{
Dynamic Companion Harmonic Circuit Models for Analysis of Power Systems with Embedded Power Electronics Devices
}

\author{
H. Garcia \\ Instituto Tecnológico de Morelia
}

M. Madrigal

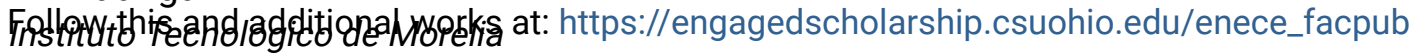

Part of the Power and Energy Commons

B. Vyakaranam

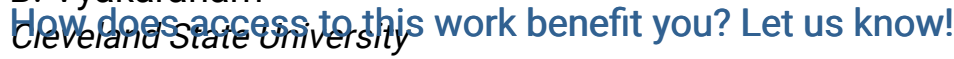

Publisher's Statement

N.diakik. this is the author's version of a work that was accepted for publication in Electric Cleveland State University

Power Systems Research. Changes resulting from the publishing process, such as peer review,

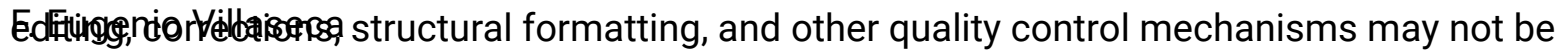

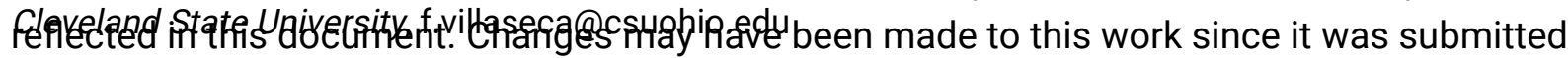

for publication. A definitive version was subsequently published in Electric Power Systems

Research, 81, 2, (02-01-2011); 10.1016/j.epsr.2010.09.010

\section{Original Citation}

H. García, M. Madrigal, B. Vyakaranam, R. Rarick and F. E. Villaseca, "Dynamic companion harmonic circuit models for analysis of power systems with embedded power electronics devices," Electr. Power Syst.

Res., vol. 81, pp. 340-346, 2, 2011.

\section{Repository Citation}

Garcia, H.; Madrigal, M.; Vyakaranam, B.; Rarick, R.; and Villaseca, F. Eugenio, "Dynamic Companion Harmonic

Circuit Models for Analysis of Power Systems with Embedded Power Electronics Devices" (2011). Electrical

Engineering \& Computer Science Faculty Publications. 214.

https://engagedscholarship.csuohio.edu/enece_facpub/214

This Article is brought to you for free and open access by the Electrical Engineering \& Computer Science Department at EngagedScholarship@CSU. It has been accepted for inclusion in Electrical Engineering \& Computer Science Faculty Publications by an authorized administrator of EngagedScholarship@CSU. For more information, please contact library.es@csuohio.edu. 


\title{
Dynamic companion harmonic circuit models for analysis of power systems with embedded power electronics devices
}

\author{
H. García ${ }^{\mathrm{a}, *}$, M. Madrigal ${ }^{\text {a }}$, B. Vyakaranam ${ }^{\text {b }}$, R. Rarick ${ }^{\mathrm{b}}$, F.E. Villaseca ${ }^{\text {b }}$ \\ a Programa de Graduados e Investigación en Ingeniería Eléctrica, Instituto Tecnológico de Morelia, Morelia, Mexico \\ ${ }^{\mathrm{b}}$ Department of Electrical and Computer Engineering, Cleveland State University, $\mathrm{OH}, \mathrm{USA}$
}

\section{Introduction}

Modern electric networks include power electronics in the form of flexible AC transmission system (FACTS) devices, high voltage DC (HVDC) transmission links, and custom power system (CUPS) devices. As a result several challenges have arisen in the modeling and analysis of modern electric network. This has been exacerbated by the inclusion of alternative energy sources and storage devices connected to the electric grid by means of power electronics converters. Two of the challenges are the modeling for transient and harmonic analysis in large networks. For transient analysis of electric networks, software such as Electro-Magnetic Transients Program (EMTP), PSCAD/EMTDC, and Simplorer use circuit discrete models, resulting in a general nodal circuit formulation that allows time-domain analysis [1,2]. On the other hand, the method of dynamic average models [2] is a powerful computational tool for large and small signal analysis of power systems, but a limitation of this method is that the harmonics generated by the switching elements are not considered. The DHD method has been shown to be a powerful tool for the dynamic analysis of power elements [3-10]. In this paper a methodology that extends the dynamic harmonic domain (DHD) analysis of large networks is presented. The method combines DHD analysis and discrete companion circuit modeling resulting in a powerful analytic technique called dynamic companion harmonic circuit modeling. It provides for a complete dynamic

\footnotetext{
* Corresponding author. Tel.: +52 4433171870.
}

harmonic analysis of the system, while preserving the advantages of discrete companion circuit models.

The methodology is illustrated by its application to a three-node power system, where the reactive power is compensated by a fixedcapacitor, thyristor-controlled reactor and its control system which controls the firing angle of the thyristors. Although the DHD method solves the differential equations of the system for the harmonics over time, the proposed method incorporates discrete companion circuit modeling $[11,12]$ into the DHD method and transforms the differential equations into algebraic equations. The discrete models obtained in this manner are admittance matrices instead of purely resistive elements, which is the case when time-domain analysis is used. Also, a control system for the voltage control of the FC-TCR is included.

\subsection{FC-TCR considerations}

A common FACTS device is the static VAR compensator, and its most important property is its ability to maintain an approximately constant voltage at its terminals by continuous adjustments of the reactive power that it exchanges with the power system. Unfortunately, this compensation is based on circuit controllers that distort the waveform of the uncompensated voltages from their ideal sinusoidal form. Nevertheless, the static compensator is commonly used and studied assuming sinusoidal voltages.

Several papers have proposed solutions for the harmonic distortion caused by thyristor-controlled reactors (TCRs). A multiphase harmonic load flow has been described in Ref. [13], and a frequency 
domain TCR model is presented in Ref. [14]. In these papers, the control of voltage is achieved by an iterative scheme of adjusting the firing angle based on harmonic analysis that leads to steady state operation. Also, a steady state analysis based on the use of harmonic domain admittance matrices and functions is presented in Ref. [15]. In Ref. [5] the dynamic harmonic behavior of the system is presented, but the control action of the compensator is not considered.

\section{Dynamic harmonic domain}

The DHD methodology is based on orthogonal bases, complex Fourier series, matrix differential operators, and the approximation of operators. The main idea underlying the DHD is that a function $x(t)$ can be approximated by the time-dependent complex Fourier series [3-6]:

$x(t)=\sum_{h=-\infty}^{h=\infty} X_{h}(t) \mathrm{e}^{j h \omega_{0} t}$

The complex Fourier coefficients $X_{h}(t)$ are a function of time. Each coefficient is calculated using:

$X_{h}(t)=\frac{1}{T} \int_{t}^{t+T} x(\tau) \mathrm{e}^{-j h \omega_{0} \tau} d \tau$

where $\omega_{0}=2 \pi / T, \tau \in[t, t+T]$ and $T$ is the period of time under consideration. Eq. (2) gives the time-evolution of the harmonics as a window of length $T$ slides over the waveform $x(t)$. In Refs. [3-6] it is shown that the state-space equation $d x(t) / d t=a(t) x(t)+b(t) u(t)$ can be represented in the DHD by

$\frac{d \mathbf{X}(t)}{d t}=[\mathbf{A}(t)-\mathbf{D}] \mathbf{X}(t)+\mathbf{B}(t) \mathbf{U}(t)$

The vector $\mathbf{X}(t)$ is the state variable whose components are the harmonics coefficients of $x(t)$; the vector $\mathbf{U}(t)$ is the system input whose components are the harmonic coefficients of $u(t)$. The matrices $\mathbf{A}(t)$ and $\mathbf{B}(t)$ are Toeplitz matrices whose elements are the harmonic coefficients of $a(t)$ and $b(t)$, respectively. The matrix $\mathbf{D}$ is a differential operator. The expression in (3) gives the dynamic evolution of the harmonic coefficients of the periodic variable $x(t)$ Also, the steady state response of (3) is obtained when the derivative of the state variable is equal to zero, that is, when $d \mathbf{X}(t) / d t=0$. In steady state the explicit dependence on time in (3) can be suppressed yielding $0=[\mathbf{A}-\mathbf{D}] \mathbf{X}+\mathbf{B U}$ or

$\mathbf{X}=-[\mathbf{A}-\mathbf{D}]^{-1} \mathbf{B U}$

Eqs. (3) and (4) are the basis of dynamic harmonic domain analysis. Eq. (3) gives the evolution in time of the harmonics of the system during the transient period and (4) establishes the condition for steady state. The steady state solution from (4) can be used as an initial condition when solving (3). This characteristic is very important since in many cases it is difficult to obtain a steady state solution of a dynamic system.

\section{Companion harmonic circuit models}

The solution of the differential equation representing an ideal inductor or capacitor can be approximated by applying the trapezoidal rule for integration to the equation. The difference equation which results can then be represented by a Norton equivalent circuit comprised of an admittance and a current source. The equivalent circuit is called the associated discrete circuit model or companion circuit model of the circuit element. Each of the inductive or capacitive elements in the electrical network of a power

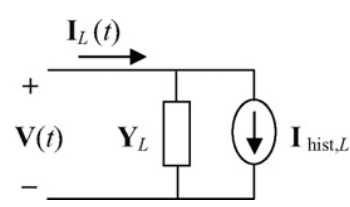

Fig. 1. Companion harmonic circuit model of an ideal inductor

system can be converted to its companion circuit model before setting up the equations which describe the entire network $[11,12]$. The companion circuit model approach can be combined with the powerful methodology of dynamic harmonic domain analysis for transient and steady state calculations in the harmonic domain. It should be noted that a companion circuit model can be derived from any implicit numerical integration formula associated with a circuit element or device [11,12]. In this section, we shall derive the companion harmonic circuit models for the inductors, capacitors, and a thyristor-controlled reactor using their defining differential equations in the DHD.

\subsection{Companion harmonic circuit modeling}

The differential equation that represents the terminal characteristics of an ideal inductor $L$ is given by

$v(t)=L \frac{d i_{L}(t)}{d t}$

According to Eq. (3), Eq. (5) may be expressed in the DHD by

$\frac{d \mathbf{I}_{L}(t)}{d t}=-\mathbf{D} \mathbf{I}_{L}(t)+\frac{1}{L} \mathbf{V}(t)$

Applying the trapezoidal numerical integration approximation to Eq. (6), the following expression is obtained:

$\frac{\mathbf{I}_{L}(t)-\mathbf{I}_{L}(t-\Delta t)}{\Delta t}=-\mathbf{D} \frac{\mathbf{I}_{L}(t)+\mathbf{I}_{L}(t-\Delta t)}{2}+\frac{1}{L} \frac{\mathbf{V}(t)+\mathbf{V}(t-\Delta t)}{2}$

where $\Delta t$ is the integration time step. Solving for $\mathbf{I}_{L}(t)$ gives the companion harmonic circuit model in the Norton equivalent form:

$\mathbf{I}_{L}(t)=\mathbf{Y}_{L} \mathbf{V}(t)+\mathbf{I}_{\text {hist }, L}$

where $\mathbf{Y}_{L}$ is a complex admittance matrix given by

$\mathbf{Y}_{L}=\frac{\Delta t}{L}\left[2 \mathbf{U}_{I}+\Delta t \mathbf{D}\right]^{-1}$

$\mathbf{U}_{I}$ is the identity matrix, and the history term $\mathbf{I}_{\text {hist }, L}$ is given by

$\mathbf{I}_{\text {hist }, L}=\frac{L}{\Delta t} \mathbf{Y}_{L}\left[2 \mathbf{U}_{I}-\Delta t \mathbf{D}\right] \mathbf{I}_{L}(t-\Delta t)+\mathbf{Y}_{L} \mathbf{V}(t-\Delta t)$

Eq. (8) is the companion harmonic circuit model of the inductor shown in Fig. 1. This model is required for the transient analysis of the system.

For a capacitance $C$, the governing differential equation is

$i_{C}(t)=C \frac{d v(t)}{d t}$

In the DHD this equation is represented by

$\frac{d \mathbf{V}(t)}{d t}=-\mathbf{D V}(t)+\frac{1}{C} \mathbf{I}_{C}(t)$

The Norton equivalent circuit for a capacitor is determined in a manner similar to that used for an inductor and is given by

$\mathbf{I}_{C}(t)=\mathbf{Y}_{C} \mathbf{V}(t)+\mathbf{I}_{\text {hist }, C}$

where

$\mathbf{Y}_{C}=C\left[\frac{2 \mathbf{U}_{I}}{\Delta t}+\mathbf{D}\right]$ 


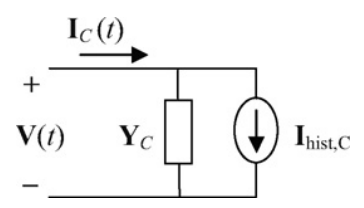

Fig. 2. Companion harmonic circuit model of an ideal capacitor.

and the history term is given by

$\mathbf{I}_{\text {hist }, C}=-\mathbf{I}_{C}(t-\Delta t)-C\left[\frac{2 \mathbf{U}_{I}}{t}-\mathbf{D}\right] \mathbf{V}(t-\Delta t)$

Eq. (13) is the companion harmonic circuit model of the capacitor given by the circuit shown in Fig. 2 .

The TCR operation is shown in Fig. 3. In this figure, the switching function $s(t)$ represents the operation of the thyristor; it is a function of the gating delay angle $\alpha$ and the conducting angle $\sigma$ for each thyristor. This function is well explained in Ref. [7].

When the thyristor is conducting, that is, $s(t)=1$, the voltage across the reactor is $v_{R}(t)$, and the current response is given by $i_{T C R}(t)$ as shown in Fig. 3. Thus, the operation of the TCR can be represented as follows. The voltage across the reactor can be described by

$v_{R}(t)=s(t) v_{T C R}(t)$

Since the voltage in the reactor is given by

$v_{R}(t)=L \frac{d i_{T C R}(t)}{d t}$

Then, substitution of Eq. (17) in (16) yields:

$\frac{d i_{T C R}(t)}{d t}=\frac{1}{L} s(t) v_{T C R}(t)$

In the DHD, Eq. (18) is represented by

$\frac{d \mathbf{I}_{T C R}(t)}{d t}=-\mathbf{D} \mathbf{I}_{T C R}(t)+\frac{1}{L} \mathbf{S}(t) \mathbf{V}_{T C R}(t)$

Then, the companion harmonic circuit model for the TCR is given by the Norton equivalent:

$\mathbf{I}_{T C R}(t)=\mathbf{Y}_{T C R}(t) \mathbf{V}_{T C R}(t)+\mathbf{I}_{\text {hist }, T C R}$

where $\mathbf{Y}_{T C R}(t)$ is a complex admittance matrix given by

$\mathbf{Y}_{T C R}(t)=\frac{t}{L}\left[2 \mathbf{U}_{I}+\Delta t \mathbf{D}\right]^{-1} S(t)$

The matrix $\mathbf{Y}_{T C R}(t)$ is a time dependent matrix because the TCR admittance depends on the state of the switching matrix function $\mathbf{S}(t)$, which depends on the power system conditions. The history
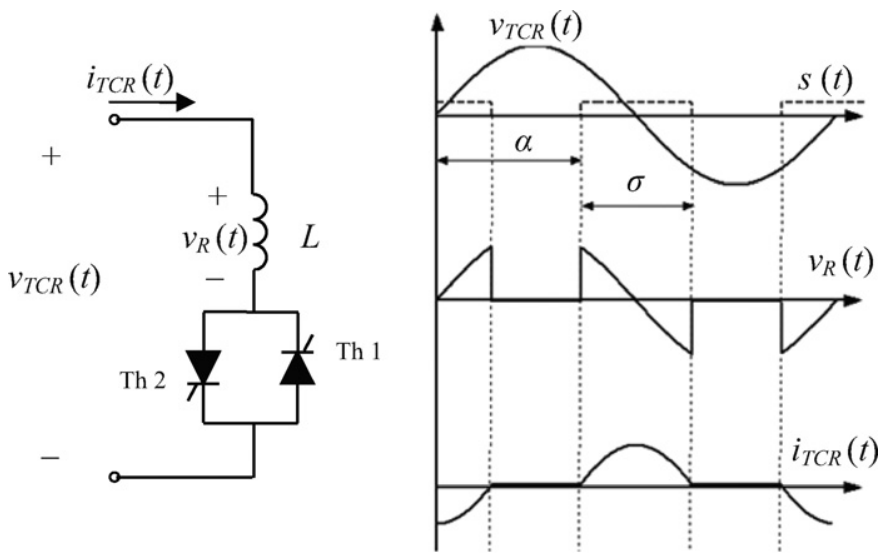

Fig. 3. Elementary thyristor-controlled reactor

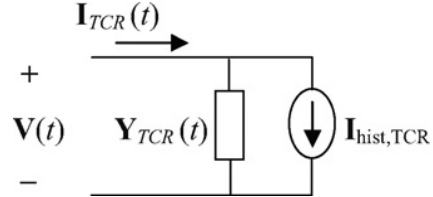

Fig. 4. Companion harmonic circuit model of a TCR.

term $\mathbf{I}_{\text {hist, } T C R}$ is given by

$$
\begin{aligned}
\mathbf{I}_{\text {hist }, T C R}= & \frac{L}{\Delta t} S^{-1}(t-\Delta t) \mathbf{Y}_{T C R}(t-\Delta t)\left[2 \mathbf{U}_{I}-\Delta t \mathbf{D}\right] \mathbf{I}_{T C R}(t-\Delta t) \\
& +\mathbf{Y}_{T C R}(t-\Delta t) \mathbf{V}_{T C R}(t-\Delta t)
\end{aligned}
$$

Fig. 4 shows the companion harmonic circuit model for transient analysis of the TCR.

\subsection{Steady state in companion harmonic circuit models}

One of the advantages of the dynamic harmonic domain method is the ability to obtain the exact steady state response of the dynamic system using Eq. (4). The steady state response for an inductor is given when the derivative of the state variable in Eq. (6) is equal to zero, that is, $d \mathbf{I}_{L}(t) / d t=0$. Then

$\mathbf{I}_{L}=\mathbf{Y}_{L, S S} \mathbf{V}$

where $\mathbf{Y}_{L, S S}=[L \mathbf{D}]^{-1}$ is a constant complex admittance matrix. It should be noted that in steady state the harmonics coefficients of the state variable vector are constant, so the time dependence can be deleted from the steady state equation (23). The exact steady state response for the capacitor is given when $d \mathbf{V}(t) / d t=0$ in Eq. (12), that is

$\mathbf{I}_{C}=\mathbf{Y}_{C, S S} \mathbf{V}$

where the constant complex admittance matrix is given by $\mathbf{Y}_{C, S S}=C \mathbf{D}$. The exact steady state response for the TCR is given when $d \mathbf{I}_{T C R}(t) / d t=0$ in Eq. (19). Then

$\mathbf{I}_{T C R}=\mathbf{Y}_{T C R, S S} \mathbf{V}_{T C R}$

where $\mathbf{Y}_{T C R, S S}=[L \mathbf{D}]^{-1} \mathbf{S}$ is a constant complex admittance matrix, and the matrix $\mathbf{S}$ is a function of the TCR terminals voltage depending on gating delay angle $\alpha$ [7]. The admittance matrix in Eq. (25) is obtained by an iterative process. With suitable initial conditions this iterative process, requires no more that 5 iterations. However, when the TCR is connected to a network which contains a high level of voltage waveform distortion, a convergence problem may arise. In many cases this problem can be solved by keeping the gating delay angle $\alpha$ constant for a short time until the voltage at the terminals of the TCR reaches a steady value.

\subsection{PI control system for the FC-TCR}

In order to maintain the voltage level at the reactive power compensator terminals at a given reference voltage, the control system shown in Fig. 5 is used. The reference voltage is $V_{\text {ref }}$, and $V_{\text {measure }}$ is the voltage at terminals of the compensator. The state-space equation for the control is given by

$\frac{d \Delta \alpha}{d t}=-\frac{1}{T} \alpha+\frac{K}{T} \varepsilon$

where $\varepsilon=V_{\text {ref }}-V_{\text {measure }}$ and $\alpha=\Delta \alpha+\alpha_{\text {hist }}$.

According to previous sections, the discrete model for the control is given by

$\alpha(t)=\frac{K \Delta t}{2 T+\Delta t} \varepsilon(t)+\alpha_{\text {hist }}$ 


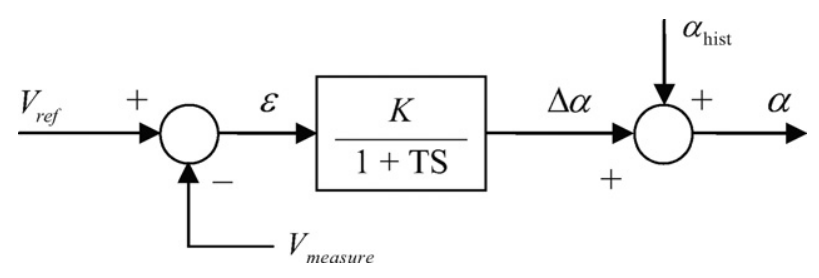

Fig. 5. PI control system.

where $\alpha_{\text {hist }}=\frac{2 T-\Delta t}{2 T+\Delta t} \alpha(t-\Delta t)+\frac{K \Delta t}{2 T+\Delta t} \varepsilon(t-\Delta t)$.

\section{Network equations formulation}

For a network with $n$ nodes, a system of $n$ equations can be derived from their companion circuit models [12]. The equation that represents the network is given by the nodal equation

$\mathbf{Y V}=\mathbf{I}-\mathbf{I}_{\text {hist }}$

where $\mathbf{Y}=n \times n$ symmetric nodal admittance matrix. $\mathbf{V}=$ vector of $n$ node voltages. $\mathbf{I}=$ vector of $n$ current sources. $\mathbf{I}_{\text {hist }}=$ vector of $n$ current history terms.

Eq. (28) is partitioned into a set $A$ of nodes with unknown voltages, and a set $B$ of nodes with known voltages. The unknown voltages are then determined by solving the system of linear algebraic equations given by

$\mathbf{Y}_{A A} \mathbf{V}_{A}=\mathbf{I}_{A}-\mathbf{I}_{\text {hist }, A}-\mathbf{Y}_{A B} \mathbf{V}_{B}$

See Ref. [11] for a detailed computational solution. In electric networks, a radial or mesh configuration can be represented directly by the admittance matrix and the known voltages $\mathbf{V}_{B}$. These voltages can be either transmission systems voltages or voltages at the power stations in distribution systems.

\subsection{Computational method to obtain and solve the nodal equation}

The computational method to solve (28) is addressed in Ref. [11]. Eq. (28) has the general form of the nodal equation given by $\mathbf{Y}_{n} \mathbf{V}_{n}=\mathbf{J}_{n}$, where $\mathbf{Y}_{n}$ is the nodal-admittance matrix defined by $\mathbf{Y}_{n}=\mathbf{A} \mathbf{Y}_{b} \mathbf{A}^{t}$. $\mathbf{A}$ is the incidence-matrix, and $\mathbf{Y}_{b}$ the branchadmittance. $\mathbf{V}_{n}$ is the nodal voltage vector, and $\mathbf{J}_{n}$ is the nodal current vector which is defined by $\mathbf{J}_{n}=\mathbf{A}\left(\mathbf{J}-\mathbf{Y}_{b} \mathbf{E}\right)$, where $\mathbf{J}$ is the branch-current source vector and $\mathbf{E}$ is the branch-voltage source vector.

The matrices and vectors $\mathbf{A}, \mathbf{Y}_{b}, \mathbf{J}$ and $\mathbf{E}$ are obtained from the voltage and current sources, branch elements and their respective connection nodes. A simple algorithm is used to automatically generate the nodal equation given by (28), from $\mathbf{Y}_{n}$ and $\mathbf{J}_{n}$, and the same algorithm came modified slightly to solve (29):

The computational procedure to solve (29) is as follows: matrices $\mathbf{Y}_{A A}$ and $\mathbf{Y}_{A B}$ are constructed and $\mathbf{Y}_{A A}$ is triangularized with ordered elimination and exploitation of sparsity. At each time step, the vector on the right-hand side of Eq. (29) is assembled from known history terms, currents and voltages. Then the system of linear equations is solved for $\mathbf{V}_{A}$, using the information contained in the triangularized admittance matrix. Before proceeding to the next time step, the history terms are updated. The initial conditions are included in the history terms for the first iteration; these can be steady state initial conditions or any other suitable initial condition. For the steady state case, the solution of the Eq. (29) does not require iteration.

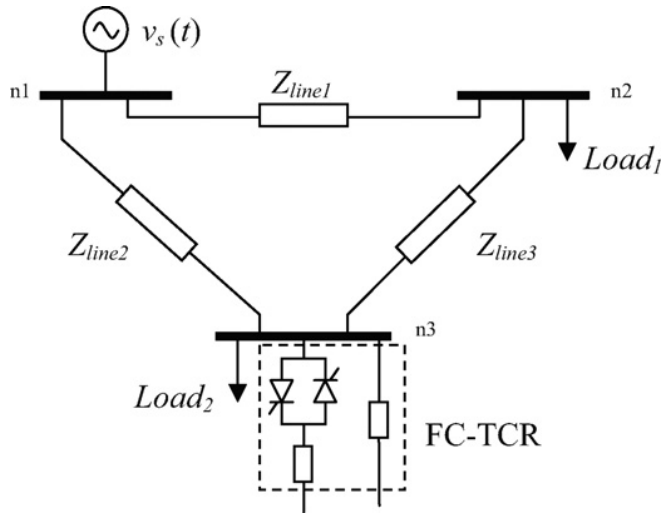

Fig. 6. Compensated power system.

\section{Numerical example}

To illustrate the application of the developed models, the $230 \mathrm{kV}-100$ MVA power system shown in Fig. 6 was simulated. The following values were used:

$$
\begin{array}{ll}
v_{s}(t)=230 \cos \omega t \mathrm{kV} & \operatorname{Load}_{1}=80+j 60 \mathrm{MVA} \\
z_{\text {line } 1}=z_{\text {line3 }}=5.29+j 63.48 \Omega & \operatorname{Load}_{2}=50+j 40 \mathrm{MVA} \\
z_{\text {line } 2}=2.645+j 31.74 \Omega & f=60 \mathrm{~Hz}, \quad \omega=2 \pi f
\end{array}
$$

For the FC-TCR, the reactor power is 100 MVAR, and the capacitor power is 90 MVAR. The case of study consists in maintain the voltage at Bus 3 equal to the voltage at Bus 1, namely, $230 \mathrm{kV}$. The simulation starts with the steady state conditions, and then $\mathrm{Load}_{2}$ is changed to $60+j 45 \mathrm{MVA}$ at the time $2 T$ seconds. A time step of

$t=T / 100 \mathrm{~s}$ and 50 harmonics where used. For the TCR control system, good results have been obtained by using a gain $k=0.3$ and a time constant $T=0.01 \mathrm{~s}$.

\subsection{Exact steady state response}

The exact steady state condition can be obtained by using the steady state companion harmonic circuit models given by Eqs. (23)-(25) for an inductor, capacitor and TCR, respectively. The nodal equation that represents the power system is given by

$$
\begin{aligned}
& \left(\begin{array}{ll}
\mathbf{Y}_{\text {line } 1}+\mathbf{Y}_{\text {line3 }}+\mathbf{Y}_{\text {Load }_{1}} & -\mathbf{Y}_{\text {line3 }} \\
-\mathbf{Y}_{\text {line3 }} & \mathbf{Y}_{\text {line2 }}+\mathbf{Y}_{\text {line3 }}+\mathbf{Y}_{\text {Load }_{2}}+\mathbf{Y}_{T C R-F C}
\end{array}\right)\left(\begin{array}{l}
\mathbf{V}_{2} \\
\mathbf{V}_{3}
\end{array}\right) \\
& =-\left(\begin{array}{l}
-\mathbf{Y}_{\text {line1 }} \\
-\mathbf{Y}_{\text {line2 }}
\end{array}\right) \mathbf{V}_{1}
\end{aligned}
$$

where the admittance matrices for the transmission lines $\mathbf{Y}_{\text {line } 1 \text {, }}$ $\mathbf{Y}_{\text {line2 }}$ and $\mathbf{Y}_{\text {line3 }}$ are obtained from the series equivalent of the line inductance matrix $\mathbf{Y}_{L, S S}$ from Eq. (23) and the line resistance matrix given by $\mathbf{Y}_{R}=\left[R \mathbf{U}_{\mathrm{I}}\right]^{-1}$. The admittance matrices for the loads $\mathbf{Y}_{\text {Load } 1}$ and $\mathbf{Y}_{\text {Load } 2}$ are obtained from the resistance matrix $\mathbf{Y}_{\text {Load,R }}$ and the inductance matrix $\mathbf{Y}_{\text {Load }, L}$, where $R_{\text {Load }}=V^{2} / P_{\text {Load }}, X_{\text {Load }}=V^{2} / Q_{\text {Load }}$, and $R_{\text {Load }} \| X_{\text {Load }}$. For the compensator, the admittance matrix $\mathbf{Y}_{T C R-F C}$ is obtained by using Eqs. (24) and (25) for the capacitor and the TCR, respectively, in parallel connection.

It should be noted that an iterative process is necessary for the solution of the nodal equation (30), since the nodal voltages and the gating delay angle are unknown. In this iterative process, a gating delay angle $\alpha=142.29^{\circ}$ and the nodal voltages were obtained. After the voltage $\mathbf{V}_{3}$ at bus 3 has been calculated, the current in the TCR 
$\mathbf{I}_{T C R}$ can be obtained.

$\mathbf{V}_{3}=\left(\begin{array}{l}\vdots \\ 2060+j 600 \\ 0 \\ 114800+j 4880 \\ 0 \\ 114800-j 4880 \\ 0 \\ 2060-j 600 \\ \vdots\end{array}\right) \quad \mathbf{I}_{T C R}=\left(\begin{array}{l}\vdots \\ 1.6506-j 18.2948 \\ 0 \\ -0.9052+j 28.0000 \\ 0 \\ -0.9052-j 28.0000 \\ 0 \\ 1.6506+j 18.2948 \\ \vdots\end{array}\right)$

\subsection{Initial value conditions}

The steady state values obtained from the solution of (30) are used for the initial conditions in the companion harmonic circuit models. Note that these steady state values could also be used in time domain simulations to initiate the simulations. In that case, the steady state initial values for this example are given by

$$
\begin{aligned}
& v_{2}(0)=\underbrace{\infty}_{\substack{h=-\infty \\
\infty}} V_{3_{h}}=227.29 \mathrm{kV} \\
& v_{3}(0)=\sum_{\substack{h=-\infty \\
3_{h}}} V_{3}=243.66 \mathrm{kV} \\
& i_{T C R}(0)=I_{T C R_{h}}=120.12 \mathrm{~A}
\end{aligned}
$$

where $h$ is the index of the harmonic component.

\subsection{Dynamic analysis using the steady state as the initial condition}

The case of study is carried out using the companion harmonic circuit models developed in Section 3.1. The nodal equation that represents the power system for dynamic analysis is

$$
\begin{aligned}
& \begin{array}{lll}
\mathbf{Y}_{\text {line1 } 1}+\mathbf{Y}_{\text {line3 }}+\mathbf{Y}_{\text {Load }_{1}} & -\mathbf{Y}_{\text {line3 }} & \mathbf{V}_{2} \\
-\mathbf{Y}_{\text {line3 }} & \mathbf{Y}_{\text {line2 }}+\mathbf{Y}_{\text {line3 }}+\mathbf{Y}_{\text {Load }_{2}}+\mathbf{Y}_{\mathrm{TCR}-F C} & \mathbf{V}_{3}
\end{array} \\
& =\begin{array}{c}
\mathbf{I}_{\text {hist, line } 1}-\mathbf{I}_{\text {hist, } \text { line3 }}-\mathbf{I}_{\text {hist }, \text { Load }_{1}} \\
\mathbf{I}_{\text {hist }, \text { line2 }}+\mathbf{I}_{\text {hist, } \text { line3 }}-\mathbf{I}_{\text {hist }, \text { Load }_{2}}-\mathbf{I}_{\text {hist }, T C R-F C}
\end{array}+\begin{array}{l}
\mathbf{Y}_{\text {line1 } 1} \\
\mathbf{Y}_{\text {line2 }}
\end{array}
\end{aligned}
$$

where the admittance matrices are obtained in a manner similar to that used in the steady state case. But in this case the equations used were Eqs. (9), (14), and (21) for the inductor, capacitor and the TCR, respectively. The current sources are given by the history terms. It should be noted that the admittance matrix for the TCR is the only one with Toeplitz structure and change every time step as a function of the gating delay angle.

The solution at every time step of the algebraic nodal equation given by (31) yields the dynamic harmonic evolution for voltages and currents of the power system. Figs. 7 and 8 show the dynamic behavior of the peak values of the harmonics for the TCR current $\mathbf{I}_{T C R}$ and the nodal voltage $\mathbf{V}_{3}$, respectively. Those figures clearly show that the simulation starts in steady state and the transient behavior of the harmonics after the load disturbance has been applied.

In Fig. 7 it is clear that the fundamental and the 3th harmonic of the current are reduced but not the 5th harmonic, which increases. This change in the magnitude of the harmonics depends on the TCR gating delay angle. When the load changes, the TCR is controlled so as to maintain the voltage at Bus 3 as shown in Fig. 8.

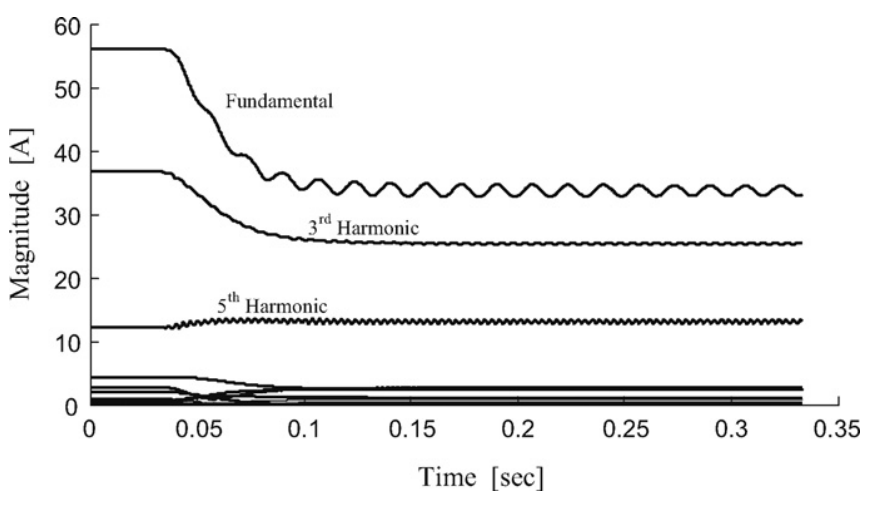

Fig. 7. Harmonic behavior of TCR current.

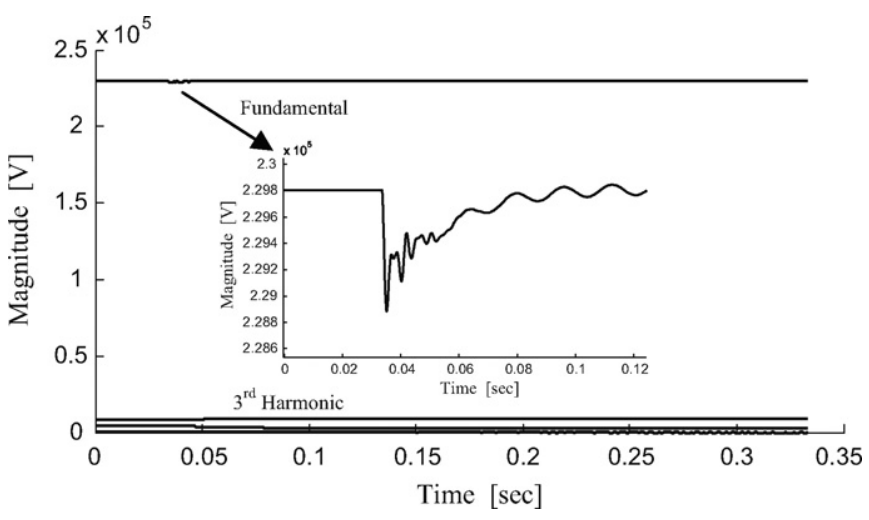

Fig. 8. Harmonic behavior of voltage at bus 3 .

Fig. 9 shows the delay angle $\alpha$ of the control system in Fig. 5 for a step increase in $\mathrm{Load}_{2}$. As expected, the delay angle increases to compensate for the increased load. Fig. 10 shows the reactive power injected into the system by the FC-TCR. Finally, Fig. 11 shows the bus voltages of the power system.

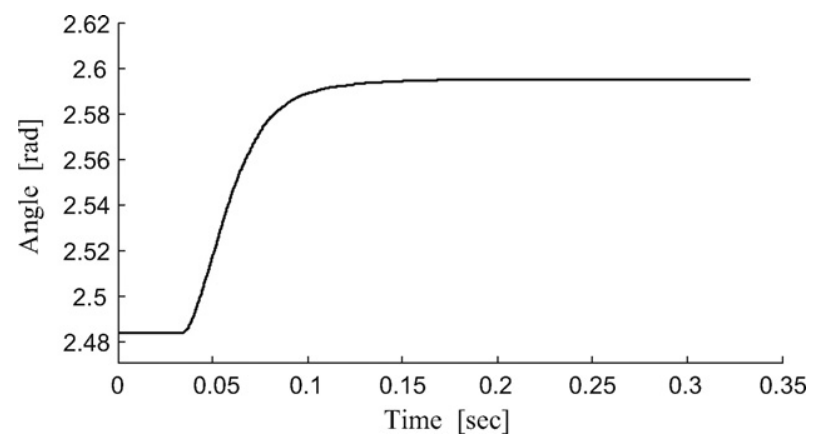

Fig. 9. Gating delay angle.

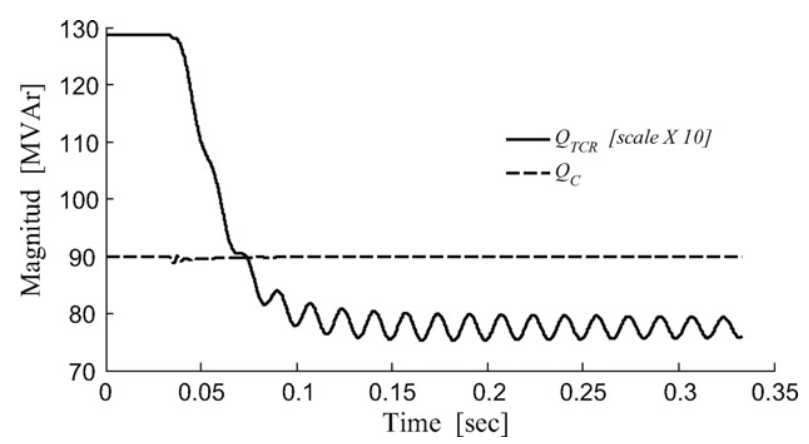

Fig. 10. Reactive power injected by the FC-TCR. 


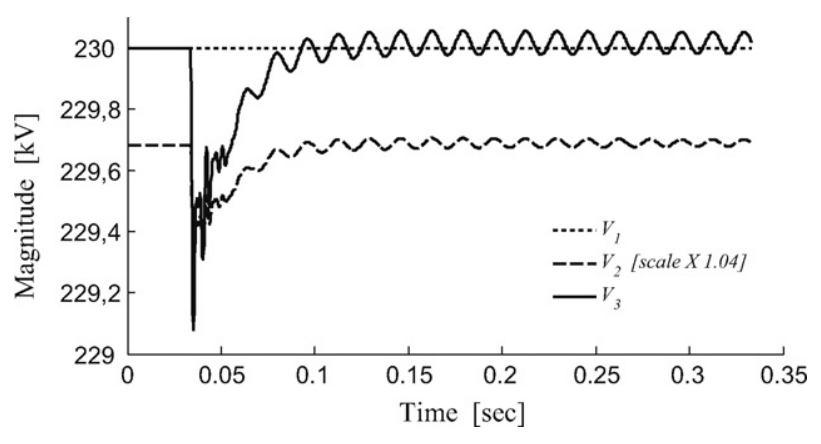

Fig. 11. Power system nodal voltages.

When an increase in the load occurs, the compensator injects reactive power into the system in order to maintain a constant voltage at Bus 3. The reactive power absorbed by the TCR decreases, while the reactive power at the capacitor remains constant as shown in Fig. 10. The voltage behavior is shown in Fig. 11. The transient period of the voltage can be observed, and the voltage returns approximately to the reference level with slight oscillations.

Using Eq. (1) the currents in the FC-TCR, which are represented in the harmonic domain, can be transformed into the time domain, and the results are shown in Fig. 12. Comparing Figs. 7 and 12, it can be seen that in the harmonic domain, the change in magnitude of the TCR current during the transient period is clearly observable; whereas in the time domain, it is impossible to observe most of the transient behavior.

A major advantage of the harmonic models developed above is that the dynamic behavior of the harmonics is available for the design of different control schemes and to analyze the resonant behavior of the power system.

\subsection{Computing time}

The computing time required to solve a $n$-nodes electrical network is given for the time required to solve the resulting $n$ simultaneous linear equations, which are of the form $\mathbf{A}_{n \times n} \mathbf{x}_{n}=\mathbf{b}_{n}$, as in Eq. (28). These are solved at each time step of simulation. This set of linear equations, when written in the harmonics domain, results in a set of $n(2 h+1)$ simultaneous linear equations which are also solved at each time step. The solutions of an $n$-nodes network, using Gaussian elimination or LU factorization required at least $n^{3} / 3$ operations [11]. Considering this, it is expected that the same $n$-nodes electrical network will require at least $(2 h+1)^{3}$ more operations if the harmonic domain is used which increases the computing time correspondingly. This computing time can be significantly reduced if the highly sparse characteristic of the resulting matrices is taking into account and sparse-matrix techniques are used. For the example discussed, a set of 303-simultaneous linear equa-

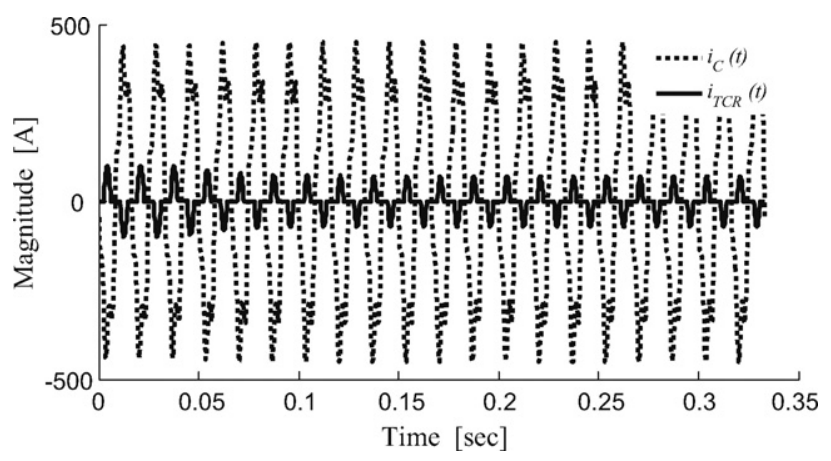

Fig. 12. Time domain representation of the currents in the FC-TCR. tions must be solved at each time step. The simulations were carried out using Matlab on an Acer Ferrari 1000 AMD Turion 64 X2 system using a time step of $0.1667 \mathrm{~ms}$. Each time step required $0.32 \mathrm{~s}$ without sparse-matrix techniques giving a total time of $32 \mathrm{~s}$ per cycle $(60 \mathrm{~Hz})$ of simulation. This time can be significantly reduced if fewer harmonics, a large time step, and sparse-matrix techniques are used.

\section{Conclusion}

This paper develops a new method for analyzing the transient and steady state response of a power system to a load disturbance in the presence of a thyristor-controlled reactive compensator with a fixed capacitance. The method combines two powerful analytic techniques: dynamic harmonic domain analysis and discrete companion circuit modeling. Used together, the technique is called companion harmonic circuit modeling. The technique allows a complete harmonic analysis of the system while preserving the advantages that accompany the use of associated discrete circuit models. One advantage is that the method provides a direct means of calculating both the steady state values of the harmonics and the transient response of the harmonics to a disturbance. The transient response is available from the explicit time-evolution of the harmonics that the method provides. Another advantage is that the power system can be modeled by a simple set of algebraic equations that easily can be implemented in simulation software instead of state-space equations involving derivatives. The results obtained from the dynamic behavior of the harmonics are valuable in the analysis of the stability and resonance of the system and in the analysis and design of control systems.

The use of the trapezoidal rule in the numerical integrations may cause oscillations in the numerical outputs, for example, in the voltage across an inductance after a current interruption or in the current through a capacitor after a voltage is switched. To mitigate this problem, the critical damping adjustment procedure described in Ref. [16] can be used. Another area for future study is the introduction of equivalent series resistances to the inductor and capacitor in the TCR. These resistances render the TCR more realistic and dampen the transients related to the load disturbance. The damping may also help to control the steady state oscillations. Finally, it should be possible to reduce the oscillations by using a more sophisticate control system.

From the implementation point of view, it is clear that it is not possible to interconnect conventional electric elements (time domain), represented by their discrete circuit models, with harmonic domain elements. This is because the circuit elements are represented by scalar quantities and the harmonic domain are represented by matrices. Therefore, discrete harmonic circuit models cannot be mixed with discrete circuit models implemented in software such as the EMTP. Nevertheless, it should be possible to implement discrete harmonic circuit models in EMTP's subroutines in order to obtain exact steady state initial condition, or to implement iterative hybrid methods where the linear network is modeled by discrete circuit models and the nonlinear network by discrete harmonic circuit models, taking the advantages of both discrete circuit models.

\section{References}

[1] V. Jalili-Marandi, V. Dinavahi, K. Strunz, J.A. Martinez, A. Ramirez, Interfacing techniques for transient stability and electromagnetic transient programs, IEEE Trans. Power Deliv. 24 (2009) 2385-2395.

[2] S. Chiniforoosh, J. Jatskevich, A. Yazdani, V. Sood, V. Dinavahi, J.A. Martinez, A. Ramirez, Definitions and applications of dynamic average models for analysis of power systems, IEEE Trans. Power Deliv. (2010).

[3] M. Madrigal, Modelling of Power Electronics Controllers for Harmonic Analysis in Power Systems, Ph.D. Dissertation, Univ. Glasgow, UK, 2001. 
[4] J.J. Rico, M. Madrigal, E. Acha, Dynamic harmonic evolution using the extended harmonic domain, IEEE Trans. Power Deliv. 18 (2003) 589-594.

[5] M. Madrigal, J.J. Rico, Operational matrices for the analysis of periodic dynamic systems, IEEE Trans. Power Syst. 19 (2004) 1693-1695.

[6] M. Madrigal, J.J. Rico, Analytical method for calculating steady-state initial value conditions of dynamic periodic networks with nonlinear components, T\&F Trans. Electric Power Compon. Syst. 34 (2006) 1331-1347.

[7] E. Acha, M. Madrigal, Power Systems Analysis: Computer Modelling and Analysis, John Wiley and Sons, Chichester, 2001.

[8] J.J. Chavez, A. Ramirez, Dynamic harmonic domain modeling of transients in three-phase transmission lines, IEEE Trans. Power Deliv. 23 (2008) 2294-2301.

[9] A. Ramirez, Frequency-domain computation of steady and dynamic states including nonlinear elements, IEEE Trans. Power Deliv. 24 (2009) 1609-1615.

[10] T. Noda, A. Semlyen, R. Iravani, Harmonic domain dynamic transfer function of a nonlinear time-periodic network, IEEE Trans. Power Deliv. 18 (2003), 1433-1341.

[11] L.O. Chua, P.M. Lin, Computer-aided Analysis of Electronic Circuits: Algorithms and Computational Techniques, Prentice Hall, New Jersey, 1975.

[12] H.W. Dommel, Electro-Magnetic Transients Program (EMTP) Theory Book, Bonneville Power Administration, Portland, 1986.

[13] W. Xu, J.R. Martí, H.W. Dommel, A multiphase harmonic load flow solution technique, IEEE Trans. Power Syst. 6 (1991) 174-182.

[14] J.G. Mayordomo, M. Izzeddine, L. Zabala, A contribution for modeling static var compensators in iterative harmonic analysis, in: IEEE/PES Proceedings ICHQP, vol. 1, Athens, Greece, 1998, pp. 285-291.

[15] L.T.G. Lima, A. Semlyen, R. Iravani, Harmonic domain periodic steady state modeling of power electronics apparatus: SVC and TCSC, IEEE Trans. Power Deliv. 18 (2003) 960-967.

[16] J.R. Martí, J. Lin, Suppression of numerical oscillations in the EMTP, IEEE Trans. Power Syst. 4 (1989) 739-747.
Héctor García received his B.Sc., M.Sc., and currently a doctoral student in electrical engineering at the Instituto Tecnologico de Morelia, Mexico. His research interests are in power systems harmonics.

Manuel Madrigal obtained his B.Sc. (with honors) and M.Sc from the Instituto Tecnologico de Morelia and Universidad Autonoma de Nuevo Leon, Mexico in 1993 and 1996, respectively. And he obtained his Ph.D. from the University of Glasgow in 2001 Since 1996 he is with the Instituto Tecnologico de Morelia where he teaches and conducts research in the area of power quality and harmonic analysis in electrical networks.

Bharat Vyakaranam was born in Visakhapatnam, India, received his B.Tech. and M.Tech. degrees in electrical engineering from the Jawaharlal Nehru Technological University, India, in 2001 and 2005, respectively. He is currently pursuing the Doctor of Engineering degree in the Department of Electrical and Computer Engineering at Cleveland State University, Ohio. His research interests are harmonic analysis of FACTS and custom power devices, and power quality.

Rick Rarick received his B.S. and M.S. in electrical engineering and his M.S. in mathematics from Cleveland State University. He is currently a doctoral student and research assistant in the Department of Electrical and Computer Engineering at Cleveland State University. His research interests are in the areas of control theory and optimization.

F. Eugenio Villaseca received his B.S. from Universidad Técnica Federico Santa María, Chile, the M.S. from the University of Notre Dame, and Ph.D. from Arizona State University, all in electrical engineering. He is currently Professor of Electrical and Computer Engineering at Cleveland State University. His research interests are in the areas of high-power electronics and control. 